\title{
Eloquent Brain Areas
}

National Cancer Institute

\section{Source}

National Cancer Institute. Eloquent Brain Areas. NCI Thesaurus. Code C104262.

Areas of the brain that control speech, motor functions, and sensory processing which are required for an individual to understand and interact with others and the environment. 